\title{
O ESPAÇO DA ÉTICA E BIOÉTICA NA GRADUAÇÃO EM MEDICINA
}

\section{The teaching of ethics and bioethics in Medical Schools \\ El espacio de la ética y bioética en la graduación de Medicina}

\author{
Maria Luiza Almeida Bastos \\ Universidade de Fortaleza - UNIFOR - Fortaleza (CE) - Brasil
}

Renata Adele de Lima Nunes

Universidade de Fortaleza - UNIFOR - Fortaleza (CE) - Brasil

\section{Samuel Verter Marinho Uchôa Lopes}

Universidade de Fortaleza - UNIFOR - Fortaleza (CE) - Brasil

\section{Rosendo Freitas Amorim}

Universidade de Fortaleza - UNIFOR - Fortaleza (CE) - Brasil

\section{RESUMO}

Objetivo: Verificar como são ofertados os conteúdos sobre ética e bioética nos currículos de formação médica das Universidades do Ceará. Métodos: Trata-se de pesquisa documental, realizada em julho de 2017 abrangendo quatro Universidades que ofertam o curso de Medicina, foram elas: Universidade de Fortaleza (UNIFOR); Universidade Federal do Ceará (UFC); Universidade Estadual do Ceará (UECE) e Universidade Christus (Unichristus). A coleta dos dados se deu por meio de revisão das matrizes curriculares dos cursos, que foram obtidas através de pesquisa nos sites das universidades ou disponibilizadas pelas coordenações dos respectivos cursos. Nesses documentos, foram investigados o caráter da oferta, a carga horária e as disciplinas específicas que abordavam os conteúdos de ética e bioética, assim como se estavam presentes nos programas pedagógicos das diversas disciplinas do curso, para averiguar a transversalidade desses temas. Resultados: Após avaliação das matrizes curriculares das quatro universidades e dos projetos pedagógicos de duas instituições de ensino analisadas, observou-se que as temáticas ética e bioética foram citadas por todas como parte integrante da formação médica em seus currículos, ainda que de maneira não uniforme entre elas. Conclusão: Os conteúdos de ética e bioética estão presentes nas matrizes curriculares dos quatro cursos de medicina do Ceará, quer seja no formato de disciplinas específicas, quer seja abordando o tema em disciplinas específicas que, dentre outros assuntos, abordam também a temática ética e bioética.

Descritores: Ética; Bioética; Educação Médica; Currículo.

\section{ABSTRACT}

Objective: To assess how ethics and bioethics contents are included in the curricula of medical schools of universities in Ceará. Methods: This documentary research was carried out in July 2017 in four universities that have medical schools: University of Fortaleza (Universidade de Fortaleza - Unifor), Federal University of Ceará (Universidade Federal do Ceará - UFC), Ceará State University (Universidade Estadual do Ceará - UECE) and Christus University (Universidade Christus - Unichristus). Data were collected by reviewing the curricula of the schools provided by their coordination offices or found on the websites of the universities. The documents were analyzed in terms of the nature of the content, the number of hours and specific courses that addressed ethics and bioethics. We also checked if the contents were included in the pedagogical planning of the other courses taught in the school to check for cross-cutting themes. Results: After analyzing the curricula of the four universities and the pedagogical projects of two universities, we found that ethics and bioethics were cited in the curricula of all the institutions as an integral part of medical education, although such inclusion did not occur in a uniform manner across the universities. Conclusion: Ethics and bioethics contents are present in the curricula of medical schools in Ceará, either in specific courses or in courses that cover, among other themes, ethics and bioethics.

Descriptors: Ethics; Bioethics; Education, Medical; Curriculum. 


\section{RESUMEN}

Objetivo: Verificar como se ofrecen los contenidos de ética y bioética en los currículums de formación médica de las Universidades de Ceará. Métodos: Se trata de una investigación documental realizada en julio de 2017 en cuatro universidades que tienen el curso de Medicina: Universidad de Fortaleza (UNIFOR); Universidad Federal de Ceará (UFC); Universidad Estadual de Ceará (UECE) y Universidad Christus (Unichristus). La recogida de datos se dio con la revisión de las matrices de los currículums de los cursos a través de una investigación en las páginas web de las universidades o aquellas disponibles en las coordinaciones de los respectivos cursos. Se investigaron en los documentos el carácter de la oferta, la carga horaria y las disciplinas específicas que tenían los contenidos de ética y bioética así como si estaban en los programas pedagógicos de las diversas disciplinas del curso para verificar la transversalidad de eses temas. Resultados: Después de la evaluación de las matrices curriculares de las cuatro universidades y de los proyectos pedagógicos de dos instituciones de educación analizadas se observó que los temas ética y bioética han sido citados por todas como parte integrante de la formación médica en sus currículums aunque de manera no uniforme entre ellas. Conclusión: Los contenidos de ética y bioética están en las matrices curriculares de los cuatro cursos de medicina de Ceará en formato de disciplinas específicas o en disciplinas que incluyen también los temas de ética y bioética.

Descriptores: Ética; Bioética; Educación Médica; Curriculum.

\section{INTRODUÇÃO}

Diante dos inúmeros desafios que o futuro nos reserva, a educação surge como ferramenta indispensável no progresso da humanidade e na consolidação dos ideais de paz, liberdade e justiça social. No início de 1993, foi criada a Comissão Internacional sobre a Educação para o Século XXI, financiada pela UNESCO. O trabalho dessa comissão levou à confecção de um relatório, o Relatório Delors, elaborado no momento em que a humanidade, diante de tantos infortúnios causados por guerras, criminalidade e subdesenvolvimento, hesita entre a aceleração do processo de desenvolvimento, sem ter a possibilidade de controlá-lo, e a resignação. Esse documento tinha o propósito de revalorizar as dimensões ética e cultural da educação. Nesse sentido, fornece recursos para que cada um venha a compreender o outro em sua especificidade, além de compreender o mundo em uma busca caótica por certa unidade. Também incentiva o indivíduo a começar pela compreensão de si mesmo em uma viagem interior, permeada pela aquisição de conhecimentos, pela meditação e pelo exercício da autocrítica(1).

Nessa perspectiva, esse documento enumera os quatro aspectos fundamentais da educação ao longo da vida: aprender a conhecer, aprender a fazer, aprender a conviver e aprender a ser. Para esse relatório, a educação na universidade também deve dispor da possibilidade de se exprimir, com toda a independência e responsabilidade, acerca de problemas éticos e sociais, como uma espécie de poder intelectual, indispensável para ajudar a sociedade a refletir, compreender e agir ${ }^{(1)}$.

A obra Os Sete Saberes Necessários à Educação do Futuro (2000), pondera que o ser humano é complexo e envolve, ao mesmo tempo, os campos físico, biológico, psíquico, cultural, social e histórico. No entanto, a educação, através das disciplinas, desintegra essa natureza humana complexa, tornando impossível a compreensão do que significa ser humano. Dessa forma, faz-se necessário tornar a condição humana o objeto essencial de todo ensino, restaurando a capacidade do homem de perceber a sua identidade complexa e sua identidade comum aos outros seres humanos ${ }^{(2)}$.

A bioética surge no contexto da saúde como um campo de reflexão entre a sobrevivência humana e os valores ${ }^{(3)}$. Na perspectiva da formação médica, a ciência toma lugar de destaque e a cura é estabelecida através de métodos diagnósticos cada vez mais elaborados que, muitas vezes, distanciam os médicos das relações humanas cujo foco da ação é o paciente. É sob essa ótica que se pondera que o ensino médico não pode se ater unicamente ao cientificismo, visto que as ciências humanas e sociais se direcionam a um melhor discernimento na relação médicopaciente. A introdução da bioética na formação médica prepara o profissional para mediações de conflitos cotidianos em saúde. O falso universo da onipotência, que se instalou culturalmente entre muitos estudantes de medicina, começa a ruir, evidenciando que não é simples tomar decisões quando estão em jogo valores morais distintos ${ }^{(4)}$.

Dentro dessa perspectiva, propõem-se os seguintes questionamentos: como a ética e a bioética estão inseridas nos currículos de graduação médica? Existe transversalidade, ou seja, o tema perpassa todas as disciplinas, ou a maioria, ao longo do curso? Esses questionamentos são necessários ao se refletir sobre os intensos debates relacionados à área da saúde nos últimos anos, fatos motivados por mudanças sociais, culturais e econômicas ocorridas, bem como suas implicações na sociedade ${ }^{(5)}$. Além disso, evidencia-se a pouca habilidade do profissional 
médico em lidar com situações em que as decisões desconsideram a autonomia, as crenças e os valores do paciente $^{(4)}$. Dessa forma, os debates em bioética são necessários à formação profissional, não se restringindo somente aos aspectos técnicos.

Todas essas mudanças sociais sugerem que a formação médica seja reelaborada, alargando as discussões quanto aos princípios éticos e a valorização dos direitos humanos. Por essa razão, considera-se relevante analisar o ensino da bioética na graduação médica. Neste estudo, objetivou-se verificar como são ofertados os conteúdos sobre ética e bioética nos currículos de formação médica das universidades do Ceará.

\section{MÉTODOS}

Trata-se de pesquisa documental, realizada em julho de 2017, abrangendo quatro universidades que ofertam o curso de Medicina, foram elas: Universidade de Fortaleza (UNIFOR), Universidade Federal do Ceará (UFC), Universidade Estadual do Ceará (UECE) e Universidade Christus (Unichristus).

A coleta dos dados se deu por meio de revisão das matrizes curriculares dos cursos, que foram obtidas através de pesquisa nos sites das universidades ou disponibilizadas pelas coordenações dos respectivos cursos.

O plano pedagógico da Faculdade de Medicina da UFC e sua matriz curricular foram extraídos do site ${ }^{(6)}$ da própria Faculdade de Medicina. As matrizes curriculares da faculdade de Medicina da Unichristus e da UECE foram obtidas nos sites institucionais ${ }^{(7,8)}$. A grade curricular e o plano pedagógico do curso de medicina da UNIFOR foram disponibilizados pela coordenação do curso( ${ }^{(9)}$.

Nesses documentos, foram investigados o caráter da oferta, a carga horária e as disciplinas específicas que abordavam o conteúdo "ética e bioética", assim como, se esses conteúdos estavam presentes nos programas pedagógicos das diversas disciplinas do curso, com o intuito para averiguar a transversalidade desses temas.

Por se tratar de pesquisa documental, com fonte de dados aberta e com informações de caráter público, não foi necessária a aprovação em comitê de ética em pesquisa com seres humanos.

\section{RESULTADOS}

Após avaliação das matrizes curriculares das quatro universidades e dos projetos pedagógicos da UNIFOR e da UFC, observou-se que as temáticas ética e bioética foram citadas como parte integrante da formação médica em seus currículos, ainda que de maneira não uniforme entre elas.

Diante da análise dos currículos e da estrutura das quatro faculdades de Medicina no Ceará, destaca-se que o conteúdo de ética e bioética foi incorporado ao currículo como disciplina específica na UFC, perpassando toda a graduação, de forma transdisciplinar, do início ao internato (Tabela I). No plano pedagógico ofertado pela UFC existem disciplinas horizontais que permeiam todo o curso, trazendo conceitos de ética com foco temático no primeiro semestre e aprofundamento prático do sexto ao oitavo semestre. Porém, não se conseguiu detectar se o conteúdo de ética ofertado é referente ao código de ética profissional, que perfaz os direitos e deveres do médico, ou se existem abordagens referentes aos conceitos de ciências sociais em saúde, aspectos relacionados à saúde coletiva e toda a sua diversidade.

A grade curricular da UECE traz disciplinas específicas para o ensino da ética e bioética, como medicina legal e deontologia, ética médica e bioética, respectivamente, nos sexto e oitavo semestre. Ainda ultrapassa o conteúdo dessas disciplinas, permeando toda a graduação médica e enfatizando a abordagem ética nas relações humanas e sociais inerentes à prática médica. Além disso, abrange disciplinas de saúde coletiva, ciências sociais e saúde, exame clínico e relação médico-paciente, além de uma disciplina no internato intitulada Introdução à tanatologia, cujo escopo oferece abordagem nas concepções humanas em saúde, principalmente em situações conflituosas, ou ainda de difíceis decisões na prática médica.

A UNIFOR não destacou disciplinas específicas para o ensino de ética e bioética, no entanto, traz o foco, em seus objetivos, para que, durante a graduação médica, o aluno seja conduzido a exercer a profissão com uma postura ética e humana com o paciente, seus familiares e a sociedade. Esse conteúdo pode ser mais percebido nas disciplinas de Internato em Saúde Coletiva, que acontecem nos últimos semestres do curso.

A Unichristus também não traz em sua matriz curricular disciplinas exclusivas ao ensino de ética e bioética, mas ressalta nos objetivos do curso de formação em medicina a adoção de posturas humanísticas e éticas do futuro profissional médico com o doente e a comunidade que está sendo assistida. 
Tabela I - Distribuição de disciplinas com conteúdo de ética e bioética em suas estruturas curriculares conforme os semestres de oferta em cursos de Medicina no estado do Ceará. Fortaleza, Ceará, 2017.

\begin{tabular}{lll}
\hline Universidade & Disciplinas & Semestre de oferta \\
\hline \multirow{4}{*}{ UFC } & Ética & $\mathrm{S} 1, \mathrm{S6}, \mathrm{S7}, \mathrm{S} 8$ \\
& Atenção á saúde individual e coletiva & $\mathrm{S} 1, \mathrm{~S} 2, \mathrm{S3}, \mathrm{S7}$ \\
& Abordagem do paciente no eixo individual e coletivo & $\mathrm{S} 1, \mathrm{~S} 5, \mathrm{~S} 6, \mathrm{~S} 7, \mathrm{~S} 8$ \\
& Saúde Coletiva & $\mathrm{S} 1$ \\
\multirow{3}{*}{ UECE } & Ciências Sociais e Saúde & $\mathrm{S} 4$ \\
& Medicina legal e deontologia & $\mathrm{S} 6$ \\
& Ética e Bioética & \\
& Dramatizando a relação médico paciente & S8 \\
UNIFOR & Internato em Saúde Coletiva & internato (S9 a S12) \\
& Diversidade humana e Saúde Coletiva & internato (S9 a S12) \\
Unichristus & Não há & Optativa \\
\hline
\end{tabular}

\section{DISCUSSÃO}

A Faculdade de Medicina da UFC tem seus conteúdos essenciais curriculares obrigatórios contidos em três eixos longitudinais curriculares: Assistência Básica em Saúde; Desenvolvimento Pessoal e Aprendizagem da Prática Profissional; Fundamentos e Prática da Assistência Médica. Um quarto eixo, denominado de Atividades Complementares, organiza os conteúdos complementares do currículo, bem como as atividades extracurriculares realizadas pelos discentes ${ }^{(6)}$.

Os eixos longitudinais curriculares são compostos por eixos temáticos, em módulos sequenciais, de modo a garantir um contato do aluno com os temas de forma contínua, consistente, articulada internamente e com as outras atividades do curso, favorecendo a sua progressiva incorporação para a vida profissional, o desenvolvimento crescente da autonomia e o domínio do estudante em relação às áreas de competência.

No eixo curricular longitudinal de Desenvolvimento Pessoal e Aprendizagem da Prática Profissional, os três eixos temáticos são: profissionalismo, comunicação e ética( ${ }^{(6)}$. Nesse eixo, os alunos desenvolverão atividades dos conteúdos de história da medicina, ética médica, bioética, relações humanas, valores humanos e desenvolvimento humano; de forma a desenvolver habilidades sensoriais, reflexivas e de comunicação, favorecendo comportamentos e atitudes afirmativas e éticas, necessárias para uma prática adequada ao futuro profissional. Pode-se perceber que esta universidade concedeu à temática da ética um lugar de destaque, assumindo a posição de eixo temático. Também é preconizado que os temas sejam abordados de maneira continuada e integrada a outras disciplinas, sendo atendido o caráter de transversalidade das temáticas ética e bioética no programa desta universidade.

A matriz curricular da UECE traz em seu escopo a disciplina Ética Médica e Bioética, no oitavo semestre ${ }^{(8)}$. Além disso, outras disciplinas da grade, como saúde coletiva (no primeiro semestre), ciências sociais e saúde, exame clínico e relação médico-paciente (no quarto semestre), medicina legal e deontologia (no sexto semestre) e introdução à tanatologia (no internato, do nono ao décimo segundo semestres), também abordam temas relacionados à ética médica. Demonstra-se, neste escopo, a preocupação em colocar as disciplinas com temas sobre ética em diversos momentos ao longo do curso, sob uma perspectiva de transversalidade, porém não foi possível avaliar de que forma são abordados os temas, já que não foi disponibilizado o projeto pedagógico do curso e esse documento também não está disponível no site da instituição.

A UNIFOR considera, em seu projeto pedagógico, que, na concepção contemporânea, o modelo de ensino tem tendências que estão moldadas às necessidades de adaptação, tanto da instituição como do corpo docente e discente, às mudanças da ciência e da sociedade, que ocorrem em velocidade exponencial. Destaca, então, que há necessidade de enfoque e estratégias que se adequem mais à formação dos profissionais que irão exercer suas atividades no século atual, entre elas: a ética e a sensibilidade humana. O projeto ainda relaciona-se com metodologias que visam um maior envolvimento dos alunos na busca do conhecimento.

Tal perspectiva de inovação baseia-se em alguns documentos e recomendações relativos à educação médica mundial nas últimas décadas e, dentre eles, destacam-se: o movimento "Saúde para Todos no ano 2000" e a Declaração de Alma Ata (1978), que têm importância capital na concepção social da saúde como um direito de todos ${ }^{(9)}$. No âmbito 
mais específico da formação profissional, espera-se que o médico egresso da Unifor possa exercer a Medicina com postura ética e humanística em relação ao paciente, à família e à comunidade, observando os aspectos sociais, culturais, psicológicos e econômicos relevantes do contexto, baseados nos princípios da bioética. Pelo enfoque dado à ética como matéria fundamental na formação médica, além da utilização como referência de documentos internacionais, visando o bem-estar de todos e a valorização humana, observa-se que a matriz curricular pretende abordar o tema da ética como elemento fundamental em sua composição, ainda que não especifique de que forma aparece nas disciplinas ou qual a carga horária utilizada para alcançar os objetivos propostos.

A Faculdade de Medicina da Unichristus disponibiliza a estrutura do curso em forma de módulos anuais, denominados de séries, num total de seis séries que correspondem aos seis anos da graduação médica. Cada ano possui em sua matriz os módulos verticais e horizontais numa estratégia de integração entre as disciplinas ${ }^{(7)}$. Durante a análise dos títulos das disciplinas na matriz da Unichristus, não se conseguiu evidenciar nenhuma disciplina específica para o ensino de ética ou bioética, assim como também não foi possível identificar disciplinas cujos escopos contenham conceitos relativos às ciências humanas e ensino de bioética. No entanto, é descrito, nos objetivos gerais da graduação em Medicina da Unichristus, que a capacitação do aluno em adquirir visão humanística, postura ética e valorização da bioética no campo da Medicina(7).

Traz, ainda, em seus objetivos específicos, o desenvolvimento humano, ético e participação social. Acreditase que essas descrições referem-se ao ensino de conceitos éticos e bioéticos no programa de determinadas disciplinas, ainda que não tenha ficado claro de que forma e em que disciplinas os temas foram abordados, além de ações relativas ao contexto da saúde, o que se julga insuficiente, visto que o fortalecimento da ética e bioética como disciplina precisa ser aprimorado, principalmente na perspectiva clínica, jurídica e filosófica ${ }^{(5)}$.

Outra observação relativa ao ensino da bioética durante a graduação Médica está relacionada ao corpo docente. No site da universidade Unichristus, o corpo de professores da faculdade de medicina é composto exclusivamente de médicos, fato inadequado a um curso que tem características e atuações diversas, principalmente no cuidado humano com demandas sociais e habilidades de comunicações importantes para exercer com zelo a profissão. A interdisciplinaridade na saúde é necessária, em especial no contexto bioético, na medida em que se traz à tona questões relacionadas a discriminação, preconceitos, moralidade e tantos outros assuntos polêmicos com relevância, que extrapolam a área da saúde. A diversificação dos saberes promove debates ricos, uma melhor compreensão e, possivelmente, atitudes mais coerentes ${ }^{(4)}$.

Acredita-se que o ensino de ética e bioética são fundamentais na formação profissional do médico, já que promovem condutas empáticas, democráticas e humanas que não podem ser abandonadas ao longo da graduação e da carreira profissional(10-12). Este estudo retratou o papel dessas disciplinas no currículo das principais universidades do estado. No entanto, uma limitação da pesquisa é o fato da regionalização, já que a pesquisa ocorreu apenas em um estado do país. Ressalta-se ainda que a metodologia utilizada, a análise documental, não permite esclarecer informações quanto ao ensino dessas disciplinas, já que não se abordaram as opiniões dos coordenadores dos cursos e dos graduandos.

Assim, faz-se necessário analisar se essas propostas curriculares são suficientes para abordar os conceitos de ética e bioética diante das demandas sociais instauradas ao longo dos anos. Sugere-se priorizar disciplinas específicas nessas abordagens, com metodologias que desenvolvam no futuro profissional uma visão integral do ser humano, não somente na saúde, mas também no contexto social e cultural do qual participa.

Além disso, é imprescindível avaliar a formação complementar para os estudantes de Medicina e médicos, com participações em cursos e simpósios relacionados a ética e bioética inerentes a pratica profissional. Estas disciplinas precisam estar incluídas no modelo de educação continuada como benefício para a manutenção da boa prática profissional.

\section{CONCLUSÃO}

O conteúdo de ética e bioética está presente nas matrizes curriculares avaliadas dos quatro cursos de Medicina do Ceará, quer seja no formato de disciplinas específicas, quer seja abordando o tema em disciplinas específicas, as quais, dentre outros assuntos, abordam também a temática ética e bioética.

\section{CONFLITOS DE INTERESSE}

Não há conflitos de interesse. 


\section{REFERÊNCIAS}

1. Delors J; International Commission on Education for the Twenty-First Century. Learning: the treasure within. Report to UNESCO of the International Commission on Education for the Twenty-first Century: Highlights. [local desconhecido]: Unesco Publishing; 1996.

2. Morin E. Os setes saberes necessários à educação do futuro. [Internet]. Disponível em: http://portal.mec.gov. br/seb/arquivos/pdf/EdgarMorin.pdf

3. Carreiro NMS, Oliveira AAS. Interconexão entre direito e bioética à luz das dimensões teórica, institucional e normativa. Rev Bioét. 2013;21(1):53-61.

4. Silva J, Leão HMC, Pereira ACAC. Ensino de bioética na graduação de medicina: relato de experiência. Rev Bioét. 2013;21(2):338-43.

5. Neves WA Jr, Araújo LZS, Rego S. Ensino de bioética nas faculdades de medicina do Brasil. Rev Bioét. 2016;24(1):98-107.

6. Universidade Federal do Ceará. Projeto pedagógico do curso de medicina 2018.1. Fortaleza: UFC; 2017. Disponível em: https://si3.ufc.br/sigaa/public/curso/ppp.jsf?lc=pt_BR\&id=657470

7. Unichristus Centro Universitário Christus. Medicina: estrutura curricular. [Internet]. 2018. Disponível em: https:// unichristus.edu.br/graduacao/medicina/

8. Universidade Estadual do Ceará. Grade curricular do curso de medicina. Fortaleza: UECE.

9. Universidade de Fortaleza. Projeto político pedagógico do curso de medicina. Fortaleza (CE): Pró-Reitoria de graduação.

10. Feitosa HN, Rego S, Bataglia P, Rego G, Nunes R. Competência de juízo moral dos estudantes de medicina: um estudo piloto. Rev Bras Educ Méd. 2013;37(1):5-14.

11. Coelho RA, Rolim JMO, Machado SLN, Feitosa HN. Teaching about ethics with an interprofessional mock trial. Med Educ. 2017;51(11):1168.

12. Feitosa HN. A competência de juízo moral e as atitudes morais dos estudantes de medicina: estudo transcultural [tese]. Porto: Universidade do Porto; 2013. Disponível em: https://repositorio-aberto.up.pt/bitstream/10216/75168/2/32384. pdf

\section{Endereço para correspondência:}

Maria Luiza Almeida Bastos

Universidade de Fortaleza - UNIFOR

Programa de Pós-Graduação em Saúde Coletiva

Av. Washington Soares, 1321, Bloco S, Sala 1

Bairro: Edson Queiroz

CEP: 60.811-905 - Fortaleza - CE - Brasil

E-mail: mluiza@edu.unifor.br 